\title{
Resource Allocation Approach for Differential Doppler Reduction in NB-IoT over LEO Satellite
}

\author{
Oltjon Kodheli*, Stefano Andrenacci*, Nicola Maturo*, Symeon Chatzinotas*, Frank Zimmer ${ }^{\dagger}$ \\ ${ }^{*}$ SnT - Interdisciplinary Centre for Security, Reliability and Trust, University of Luxembourg, Luxembourg \\ Email: \{oltjon.kodheli, stefano.andrenacci, nicola.maturo, symeon.chatzinotaz\}@uni.lu \\ ${ }^{\dagger}$ SES S.A., Betzdorf, Luxembourg \\ Email: frank.zimmer@ses.com
}

\begin{abstract}
Internet of things (IoT) over satellite is an attractive system architecture which has been proposed as a key-enabling technology, to extend the coverage in remote areas (e.g. desert, ocean, forest, etc), particularly where a terrestrial network is impossible or impractical to reach. One of the most promising technologies that fit the IoT vision of low-power, wide area networks (LPWAN) is the narrowband IoT (NB-IoT). While low earth orbit (LEO) satellites are favourable because of their lower round trip time (RTT) and lower propagation loss in the communication link, they come up with a significantly increased Doppler shift. In our NB-IoT over LEO satellite architecture, we identify the problem of high differential Doppler among channels of different users on Earth, which leads to the performance degradation of our system. In this paper, we propose a resource allocation approach in order to reduce the high values of differential Doppler under the maximum value supported by the standard itself.
\end{abstract}

\section{INTRODUCTION}

By 2020, it is estimated that more than thirty billion devices will be connected through wireless communications [1]. This is due to the inclusion of machines in the telecommunication network, promoting a completely new paradigm, different from the traditional one where the core of the communication was the human-to-human $(\mathrm{H} 2 \mathrm{H})$ interaction. The new paradigm is termed as machine-to-machine (M2M) communication with its main application, the IoT [2]. IoT refers to a tremendous number of "things" able to generate and exchange information with each other, where the term "things" holds for low complexity and extremely cheap devices like sensors in smart home or city, monitoring devices in eHealth applications, connected cars etc. To this extent, IoT possesses the power to revolutionize the way we live, towards an intelligent society. The 3rd Generation Partnership Project (3GPP) set up the NB-IoT standard as part of Release 13 which is currently evolving and growing towards new releases with improved capabilities, as it is going to be a crucial application in the future 5G network [3], [4]. The key challenge of this technology, in terms of connectivity, is to provide a global and ubiquitous coverage to the IoT devices. However, in many cases, these devices are distributed in remote areas (e.g. desert, ocean, forest, etc.) where the terrestrial network does not exist or it is too impractical/cost-ineffective to reach. Furthermore, terrestrial networks are still, not capable to connect the tremendous number of IoT devices and terminals deployed all over the world. Therefore, the role of the satellite to extend and complement the terrestrial IoT network is crucial and irreplaceable.

The idea of satellite-terrestrial network integration, which historically have been developed separately from each other, was revisited while the Long Term Evolution (LTE) was standardized [5]. To this aim, the geostationary (GEO) orbit was firstly considered for an LTE-satellite integrated network. In particular, in [6]-[8], resource allocation algorithms for multicast transmissions and Transmission Control Protocol (TCP) performance were analyzed in a LTE-based GEO system, in order to deal with the GEO large delays. In [9] the air interface for massive machine type communication (mMTC) over GEO satellites was studied while in [10] new random access techniques applicable to satellite networks were treated.

Recently, 3GPP started the new radio access network (RAN) activities related to Non-Terrestrial Networks, which aims to study the challenges and possible solutions to the integration of the satellites, with different orbital architectures (e.g. GEO, MEO and LEO), in the 5G network [11], [12]. While LEO satellites can solve to a certain extent the problem of GEO large delays in the communication link, they suffer from an increased Doppler shift. Moreover, in IoT applications LEO orbits are advantageous due to their smaller propagation signal loss, which for the low complexity and cheap IoT devices is crucial to close the link-budget, as shown in [13]-[15]. A survey on satellite-based wireless sensor networks, focusing on system architectures and scenarios can be found in [16]. In [17] a Maximum A Posteriori (MAP) Doppler estimator in Broadband Mobile LEO Satellite Communications is analyzed, while in [18] and [19] GNSS based solutions for Doppler shift compensation in LTE and 5G over a LEO mega-constellation, are suggested. Moreover, in [20] a novel fast tracking Doppler compensation in OFDM-based LEO transmissions is proposed. However, apart from the high Doppler shift, which can be treated as in the above mentioned papers, there is also the problem of differential Doppler shift among users inside a coverage area. This is due to the fact that different users experience different channels based on their location and communicate with the satellite at different elevation angle. As a matter of fact, the differential part of the Doppler should be compensated at the user side. However, adding extra algorithms at the user side for LEO Doppler compensation means moving further away from the IoT vision of very cheap 


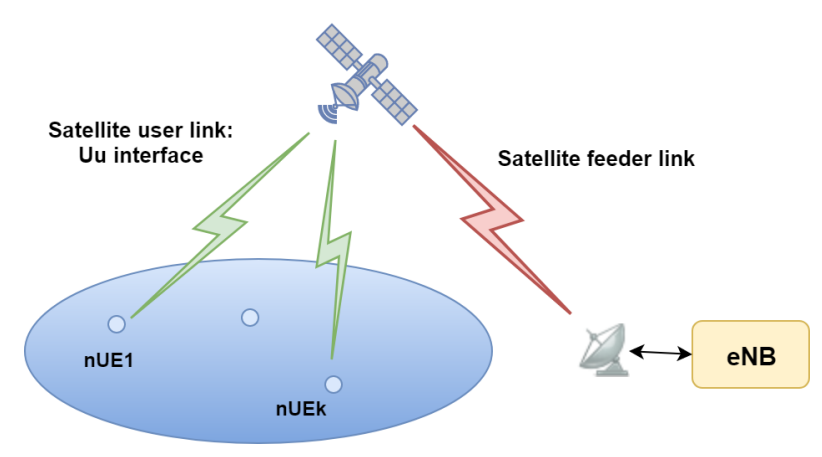

Fig. 1. NB-IoT over Satellite architecture

and low complexity devices.

Therefore, the aim of this paper is to propose a resource allocation approach in order to solve the problem of the high differential Doppler among users, while maintaining the same complexity at the user side. We consider a system architecture in which a LEO satellite provides NB-IoT services to the NBIoT user equipments (nUEs), which are placed over a fixed region on Earth isolated from a terrestrial infrastructure.

\section{SySTEM MODEL}

\section{A. System Parameters and Assumptions}

In our scenario, we consider a number of nUEs, which are directly connected to the eNB through the LEO satellite link, placed in a fixed area on Earth with diameter $D=200 \mathrm{~km}$ (see Fig. 1). This area can be covered by the satellite for a certain amount of time, depending on the altitude of the satellite, the minimum elevation angle of communication and the directivity of the antenna. In our system, we consider a LEO satellite with an altitude $h=1000 \mathrm{~km}$ and a minimum elevation angle of communication $\alpha_{\min }=45$ degrees. In addition, the following assumptions are made for the considered NBIoT over satellite architecture: i) we consider the channel between the eNB and the satellite (feeder link) as ideal. The assumption is justified by the scope of the study, which focuses on the differential Doppler shift; ii) NB-IoT air interface in the user link; iii) a standalone NB-IoT deployment with a carrier frequency $f_{c}=2 \mathrm{GHz}$; iv) the nUEs are fixed on Earth and no mobility is foreseen for them. Please note that the carrier frequency corresponds to the LTE carrier and is normally used for the in-band and guard-band deployment of the NB-IoT [21]. However, we assume that the target area covered by the satellite is isolated from the terrestrial coverage, hence we can use this carrier for our stand-alone NB-IoT deployment, since it is not yet defined by the regulators. Last but not least, the satellite is assumed to be transparent.

\section{B. Signal Model}

The channel model of a satellite-terrestrial communication link can be approximately modeled as additive white Gaussian noise channel by neglecting the multipath fading [19]. This is justified by the assumption of having perfect line of sight (LoS) transmission in a remote area. Therefore, the only component in our satellite link would be the LoS component impaired by a Doppler shift due to the movement of the satellite.

1) Downlink transmission: In the downlink, orthogonal frequency-division multiple access (OFDMA) is applied with a subcarrier spacing (SCS) of $15 \mathrm{kHz}$. One OFDM symbol contains 12 subcarriers occupying this way the whole bandwidth of $180 \mathrm{kHz}$. Seven consecutive OFDM symbols form one slot, which is represented in a resource grid form in Fig.2. Please note that this is the same resource grid for LTE standard with a normal cyclic prefix (CP) length. By summing up these slots, the subframes and radio frames are formed in the same way as in LTE.

The transmitted baseband analog signal from the eNB can be written as:

$$
s(t)=\frac{1}{\sqrt{N}} \sum_{n=0}^{N-1} a[n] e^{j 2 \pi f_{s} n t} \cdot u_{T}(t)
$$

where $a[n]$ represents the symbols (data or pilots) carried by the $n$-th subcarrier, $u_{T}(\cdot)$ is a window function which in OFDM symbol is rectangular, hence $u_{T}(t)=1$ when $0 \leq t \leq T$. The window length $T=T_{s}+T_{c p}$ where $T_{s}$ is the symbol period and $T_{c p}$ is the cyclic prefix length. $f_{s}=1 / T_{s}$ represents the subcarrier spacing. The complex symbols $a[n]$ are QPSK modulated for all the downlink physical (PHY) channels and are represented by a square in the Fig. 2 .

The received analog signal by the k-th $n U E$ after downconversion can be expressed as:

$$
\begin{aligned}
r_{n U E}^{k}(t) & =e^{j 2 \pi f_{d k}(t) t} \cdot h_{k}(t) \cdot s(t)+\omega_{k}(t) \\
& =e^{j 2 \pi f_{d c}(t) t} \cdot e^{j 2 \pi \Delta f_{d k}(t) t} \cdot h_{k}(t) \cdot s(t)+\omega_{k}(t)
\end{aligned}
$$

where $\omega_{k}(t)$ is the additive white Gaussian noise, $f_{d k}(t)$ is the Doppler shift which has a dependency on time based on the position of the LEO satellite, $h_{k}(t)$ are the channel coefficients and $s(t)$ is the transmitted analog baseband signal from the base station eNB. Please note that the Doppler shift can be written as $f_{d k}(t)=f_{d c}(t)+\Delta f_{d k}(t)$, where $f_{d c}(t)$ is the common part of Doppler shift experienced by all the nUEs in the coverage and is given by the Doppler curve of one of

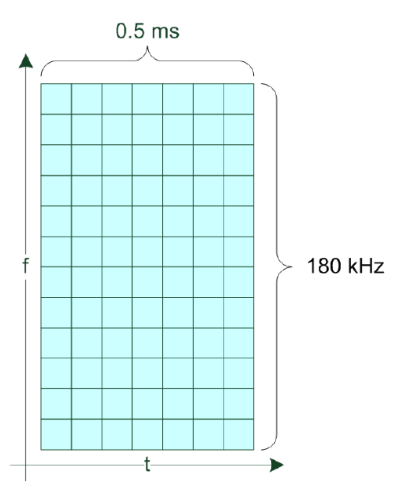

Fig. 2. Slot structure of OFDMA and SC-FDMA with $15 \mathrm{kHz}$ SCS 
the users, taken as reference. $\Delta f_{d}(t)$ is the differential part of the $\mathrm{k}$ - $t h$ nUE which has a dependency on the relative position of nUEs with respect to the reference user. From equation 1 and 2, and assuming that the frequency offset of the local oscillator is negligible, the received $l$-th symbol of the $\mathrm{k}-t h$ user of a frame transmitted at time $\left[t^{\prime}, t^{\prime}+T\right]$ after sampling at time $t=l T_{s} / N$, FFT and equalization can be written in the discrete form as:

$$
r_{l, k}=e^{j 2 \pi \epsilon_{k} l / N} \cdot a_{l, k}+\omega_{l, k}
$$

where $l=0,1, \ldots, N-1$ and $N$ is the number of subcarriers assigned to one nUE. It is worth highlighting that $\epsilon_{k}=f_{d k} / f_{s}$ is the normalized Doppler shift with respect to subcarrier spacing. This parameter also drives the performance degradation in our scenario. Please note that the Doppler shift will also have a small variation during each OFDM symbol time. However this time is so small that we assume a constant $f_{d k}$ along $\left[t^{\prime}, t^{\prime}+T\right]$.

2) Uplink transmission: In the uplink, single-carrier FDMA (SC-FDMA) signal is used. Basically, it is the same as OFDM but changes the way we map symbols into subcarriers, by applying IDFT to the complex modulated data. These data can be BPSK or QPSK modulated depending on the PHY uplink channel.

The transmitted signal by the $k$-th nUE will have the following form:

$$
s_{k}(t)=\frac{1}{\sqrt{N_{k}}} \sum_{n=0}^{N_{k}-1} b_{k}[n] e^{j 2 \pi f_{s} n t} \cdot u_{T}(t)
$$

where $b_{k}[n]$ are the symbols mapped into subcarriers after applying IDFT and $N_{k}$ is the number of subcarriers assigned for transmission for the $k$-th nUE. In the uplink, the number of subcarriers assigned for transmission can be 1 (single-tone), 3,6 or 12 (multi-tone) [22]. It is worth mentioning here that single-tone SC-FDMA can be either with a $3.75 \mathrm{kHz}$ or 15 $\mathrm{kHz}$ SCS. However, in our system we will consider only the format with a $15 \mathrm{kHz}$ SCS and one slot can be represented in the same way as in the downlink OFDMA case. The received baseband signal at the eNB is given by the superposition of each signal from nUEs

$$
\begin{aligned}
r_{e N B}(t) & =\sum_{k=1}^{M} e^{j 2 \pi f_{d k}(t) t} \cdot h_{k}(t) \cdot s_{k}(t)+\omega_{k}(t) \\
& =e^{j 2 \pi f_{d c}(t) t} \sum_{k=1}^{M} e^{j 2 \pi \Delta f_{d k}(t) t} \cdot h_{k}(t) \cdot s_{k}(t)+\omega_{k}(t)
\end{aligned}
$$

where $M$ is the number of nUEs transmitting at a certain time.

Please note that, in both transmissions, downlink and uplink, even though the Doppler shift experienced by each nUE is different, the major part of it is common for the region under study. We assume that the common part of the Doppler shift can be ideally pre-compensated in the downlink, or post compensated in the uplink at the Gateway. The Gateway knows the position of the satellite (based on the trajectory) at a certain

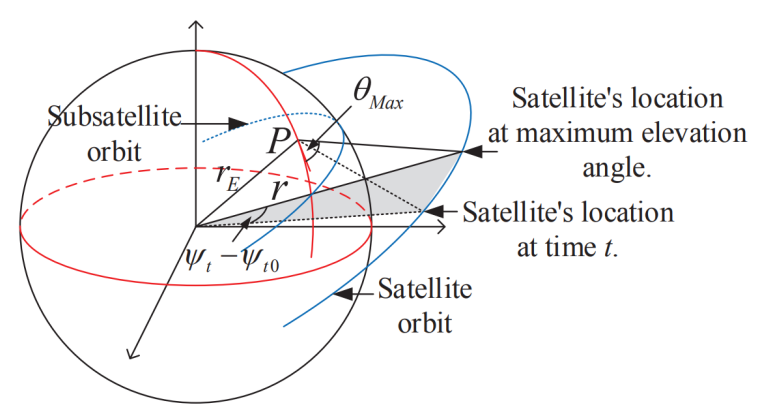

Fig. 3. Geometric representation of the LEO satellite orbit [17]

time and can calculate the common time-varying Doppler shift for the coverage region. For a more precise estimation of the position of the satellite GNSS solutions proposed in [18] can be used. As a matter of fact, by assuming perfect compensation of the common Doppler shift at the Gateway, equation (2) and equation (5) will be transformed as follows:

$$
\begin{gathered}
r_{n U E}^{k}(t)=e^{j 2 \pi \Delta f_{d_{k}}(t) t} \cdot h_{k}(t) \cdot s(t)+\omega_{k}(t) \\
r_{e N B}(t)=\sum_{k=1}^{M} e^{j 2 \pi \Delta f_{d_{k}}(t) t} \cdot h_{k}(t) \cdot s_{k}(t)+\omega_{k}(t)
\end{gathered}
$$

As a result, the parameter which causes degradation of performance in our scenario is the differential Doppler shift between nUEs and not the Doppler shift itself. At this point, it is highly important to characterize the maximum differential Doppler experienced in the coverage area, in order to evaluate the induced degradation, both in downlink and in uplink.

\section{MAXIMUM DiFFERENTIAL DOPPLER CHARACTERIZATION}

Let us start from the analytical expression of the Doppler shift at an observation point $\mathrm{P}$ on Earth. We consider a trajectory of a LEO satellite, which usually has an altitude from $500 \mathrm{~km}-2000 \mathrm{~km}$. The geometry for Doppler shift calculation is provided in Fig. 3 and a closed-form expression of the Doppler shift at a certain point $\mathrm{P}$ on Earth is provided in [23]. The formula is as follows:

$$
f_{d}(t)=-\frac{f}{c} \cdot \frac{w_{F}(t) r_{E} r \sin \left[\psi(t)-\psi\left(t_{0}\right)\right] \eta\left(\theta_{\max }\right)}{\sqrt{r_{E}^{2}+r^{2}-2 r_{E} r \cos \left[\psi(t)-\psi\left(t_{0}\right)\right] \eta\left(\theta_{\max }\right)}}
$$

where

$$
\eta\left(\theta_{\max }\right)=\cos \left[\cos ^{-1}\left(\frac{r_{E}}{r} \cos \theta_{\max }\right)-\theta_{\max }\right]
$$

$f$ is the carrier frequency, $r_{E}$ is the radius of Earth, $r$ is the orbit radius of the satellite and $\psi(t)-\psi\left(t_{0}\right)$ is the angular difference between satellite location at time $t$ and satellite location at maximum elevation angle $\theta_{\max }$ (see Fig. 3). Furthermore, $w_{F}(t)$ is the angular velocity of the satellite in the ECF (Earth central fixed) frame which can be approximated as a constant by the following expression:

$$
w_{f} \approx w_{s}+w_{E} \cos (i)
$$




$$
\begin{aligned}
\Delta f_{d \text { max }}^{x}(t) & =\left|\frac{f w_{s} r_{E}}{c}\left(\frac{r \sin \left(w_{s} t\right)-D}{\sqrt{r_{E}^{2}+r^{2}-2 r_{E} r \cos \left(w_{s} t\right)+D^{2}-2 D r \sin \left(w_{s} t\right)}}-\frac{r \sin \left(w_{s} t\right)}{\sqrt{r_{E}^{2}+r^{2}-2 r_{E} r \cos \left(w_{s} t\right)}}\right)\right| \\
\Delta f_{d \text { max }}^{y}(t) & =\left|\frac{f w_{s} r_{E} r \sin \left(w_{s} t\right)}{c}\left(\frac{\eta\left[g^{-1}\left(\frac{h}{D / 2}\right)\right]}{\sqrt{r_{E}^{2}+r^{2}-2 r_{E} r \cos \left(w_{s} t\right) \eta\left[t g^{-1}\left(\frac{h}{D / 2}\right)\right]}}-\frac{1}{\sqrt{r_{E}^{2}+r^{2}-2 r_{E} r \cos \left(w_{s} t\right)}}\right)\right|
\end{aligned}
$$

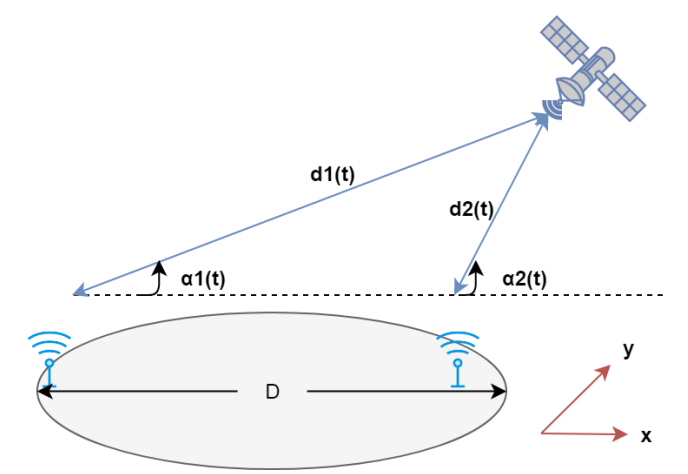

Fig. 4. Two nUE experiencing the maximum differential Doppler shift along $\mathrm{x}$-axis

$w_{s}$ is the angular velocity of the satellite in the ECI (Earth central inertial) frame, $w_{E}$ is the angular velocity of the Earth and $i$ is the satellite orbit inclination. For simplicity let us assume $w_{E}=0$ and $t=0$ the time when the satellite is at the maximum elevation angle. By taking these assumptions equation (8) will transform as follows:

$$
f_{d}(t)=-\frac{f}{c} \cdot \frac{w_{s} r_{E} r \sin \left(w_{s} t\right) \eta\left(\theta_{\max }\right)}{\sqrt{r_{E}^{2}+r^{2}-2 r_{E} r \cos \left(w_{s} t\right) \eta\left(\theta_{\max }\right)}}
$$

Having the closed-form expression of the Doppler shift as a function of time, we can now characterize the differential Doppler between two points on Earth. In order to simplify the derivation, we separate the analysis into two axes, that we will call as $x$-axis and $y$-axis. The $x$-axis represent the axis along the movement of the satellite and the $y$-axis is the one perpendicular to it.

\section{A. Along $x$-axis}

Let us now characterize the differential Doppler along the $x$ axis (please refer to Fig. 4). We are interested in characterizing the upper bound (max value) of the differential Doppler along $x$-axis with respect to time, which happens when $2 \mathrm{UE}$ are placed at the maximum distance $D$ in the coverage area on Earth and in the trajectory where the satellite reaches its maximum elevation angle $\theta_{\max }=\pi / 2$. Therefore, it can be calculated that:

$$
\eta(\pi / 2)=\cos \left[\cos ^{-1}\left(\frac{r_{E}}{r} \cos (\pi / 2)-\pi / 2\right)\right]=1
$$

By geometrical considerations, it can be easily proven that the following formulas hold as in [18]:

$$
d_{1}(t)=\sqrt{r_{E}^{2}+r^{2}-2 r_{E} r \cos \left(w_{s} t\right)}
$$

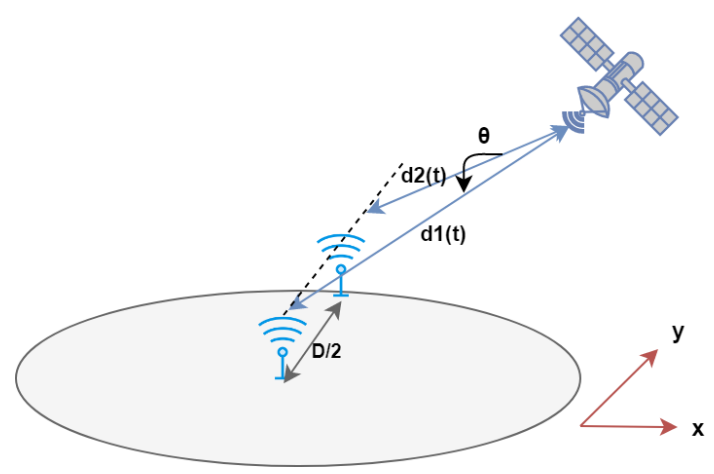

Fig. 5. Two nUE experiencing the maximum differential Doppler shift along $\mathrm{y}$-axis

$$
\cos \alpha_{1}(t)=\frac{r \sin \left(w_{s} t\right)}{\sqrt{r_{E}^{2}+r^{2}-2 r_{E} r \cos \left(w_{s} t\right)}}
$$

By substituting (15) and (16) into equation (13) we obtain the Doppler shift as a function of the elevation angle.

$$
f_{d 1}(t)=-\frac{f w_{s} r_{E} \cos \alpha_{1}(t)}{c}
$$

Finally, the differential Doppler would have the following form.

$$
\Delta f_{\text {dmax }}^{x}(t)=\left|\frac{f w_{s} r_{E}}{c} \cdot\left(\cos \alpha_{2}(t)-\cos \alpha_{1}(t)\right)\right|
$$

So now the problem of finding the differential Doppler scales down to expressing the elevation angle $\alpha_{2}$ as a function of $\alpha_{1}$. Taking into account the geometry in Fig. 4, it can be written that:

$$
\begin{gathered}
d_{1}(t) \cos \alpha_{1}(t)=d_{2}(t) \cos \alpha_{2}(t)+D \\
d_{2}(t)^{2}=d_{1}(t)^{2}+D^{2}-2 D h_{1}(t) \cos \alpha_{1}(t)
\end{gathered}
$$

Solving (19) and (20):

$$
\cos \alpha_{2}(t)=\frac{d_{1}(t) \cos \alpha_{1}(t)-D}{\sqrt{d_{1}(t)^{2}+D^{2}-2 D d_{1}(t) \cos \alpha_{1}(t)}}
$$

Finally, by plugging this expression into (18) and substituting the expressions of $d_{1}(t)$ and $\cos \alpha_{1}(t)$ we are able to obtain the closed-form equation of the maximum differential Doppler along $\mathrm{x}$-axis with respect to time (see equation (11)). 


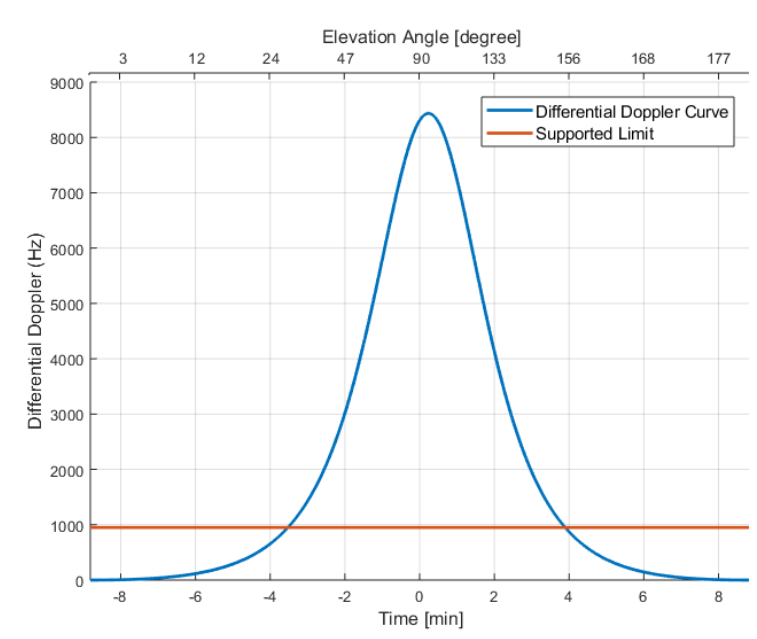

Fig. 6. Maximum differential Doppler with respect to time and satellite elevation angle along $\mathrm{x}$-axis

\section{B. Along y-axis}

The maximum differential Doppler experienced in a region with diameter $D$ on Earth along the $y$-axis is between 2 nUEs, one placed at the center of the region and the other at $D / 2$ distance in the $y$-direction. Please refer to Fig. 5 for geometry considerations. The only parameter that changes between the 2 UEs is the maximum elevation angle $\theta_{\max }$ which will be different. For the UE placed at the center of the region, the maximum elevation angle is $\theta_{1 \max }=\pi / 2$. At this point, it can be easily proved that $d 1(t)=h$, where $h=r-r_{E}$ is the altitude of the satellite. This is the moment that the other nUE also experiences the maximum elevation angle, as proved in [23]. From geometrical considerations it can be calculated that:

$$
\theta_{2 \max }=t g^{-1}\left(\frac{h}{D / 2}\right)
$$

Therefore the maximum differential Doppler can be expressed as:

$$
\Delta f_{d \max }^{y}(t)=\left|f_{d 1}\left(t, \theta_{1 \max }\right)-f_{d 2}\left(t, \theta_{2 \max }\right)\right|
$$

By plugging the values of $\theta_{1 \max }$ and $\theta_{2 \max }$ into (13) and making the difference, the final closed-form expression of the maximum differential Doppler along $y$-axis as a function of time can be obtained (see equation (12)).

\section{Problem Statement}

As we previously showed in the mathematical representation of the downlink and uplink signals, the factor causing performance degradation is the differential Doppler shift, assuming that the common Doppler shift for all the nUEs in the coverage area can be ideally pre(post)-compensated in the gateway. By plugging the system parameters of our scenario in (11) and (12) and run numerical simulations, we obtain the curves of the differential Doppler as a function of time and elevation angle of the satellite as in Fig. 6 and Fig. 7. We have shown the results for elevation angle that goes from 0 to 180 degrees.

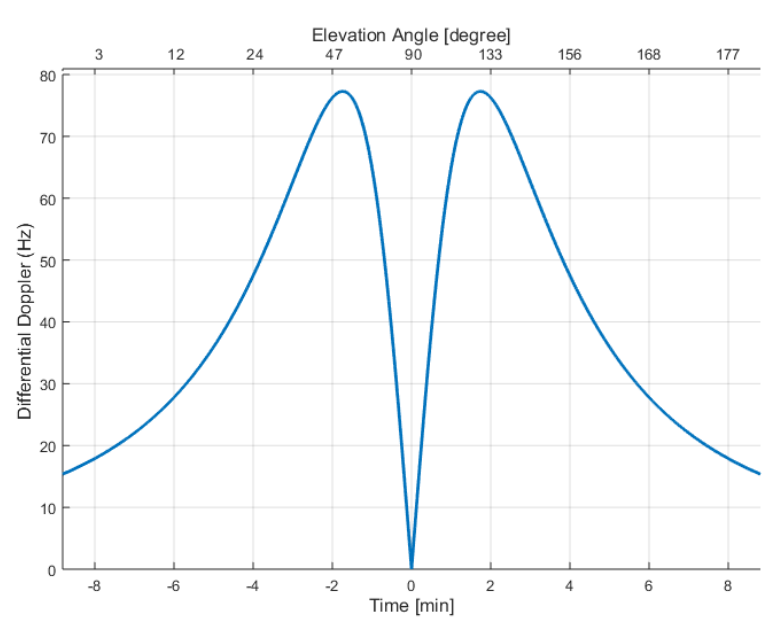

Fig. 7. Maximum differential Doppler with respect to time and satellite elevation angle along $\mathrm{y}$-axis

However, the region of interest is from 45 to 135 degrees as this is the minimum elevation angle of communication in our scenario. Finally, the differential Doppler achieves the peak values of approximately $8.5 \mathrm{kHz}$ along $x$-axis and $0.075 \mathrm{kHz}$ along $y$-axis.

In the downlink transmission, each nUE will receive the $180 \mathrm{kHz}$ NB-IoT carrier with a certain differential Doppler shift depending on user's location. All the nUEs acquire time and frequency synchronization with a cell and detect the physical layer ID through cell search procedures, which include the primary synchronization signal (NPSS) and secondary synchronization signal (NSSS) [3]. With the NPSS the nUE synchronize to the correct carrier frequency and achieve subframe, slot and symbol synchronization in the time domain. Whereas, with the NSSS the nUE is able to achieve radio frame synchronization. As a matter of fact, since the maximum value of differential Doppler $(8.5 \mathrm{kHz})$ is inside one SCS $(15 \mathrm{kHz})$ we assume that all the nUEs are able to synchronize to their shifted carrier frequency through the cell search procedure, and demodulate correctly the downlink signal.

In the uplink transmission, each nUE will generate its own signal and transmit it to the eNB. As we previously mentioned, to each nUE can be assigned $1,3,6$ or 12 subcarriers to transmit in the uplink. Due to the slotted structure of SCFDMA, all the subcarriers should be synchronized both in time and frequency, in order to avoid overlap among them. However, these subcarriers will arrive to the satellite with different Doppler shifts, negating the orthogonality in the final SC-FDMA signal. It can be calculated that the standard can support up to $950 \mathrm{~Hz}$ of Doppler shift among subcarriers. This value is derived using 3GPP specification about mobile UEs, carrier at $2 \mathrm{GHz}, 15 \mathrm{kHz} \mathrm{SCS}$ and maximum speed at 500 $\mathrm{km} / \mathrm{h}$ [24]. As LTE can support this mobility of users, it means that it can also support and mitigate a loss of orthogonality of up to $950 \mathrm{~Hz}$. Referring now to our numerical results, it can be noted that the differential Doppler is much higher than the 


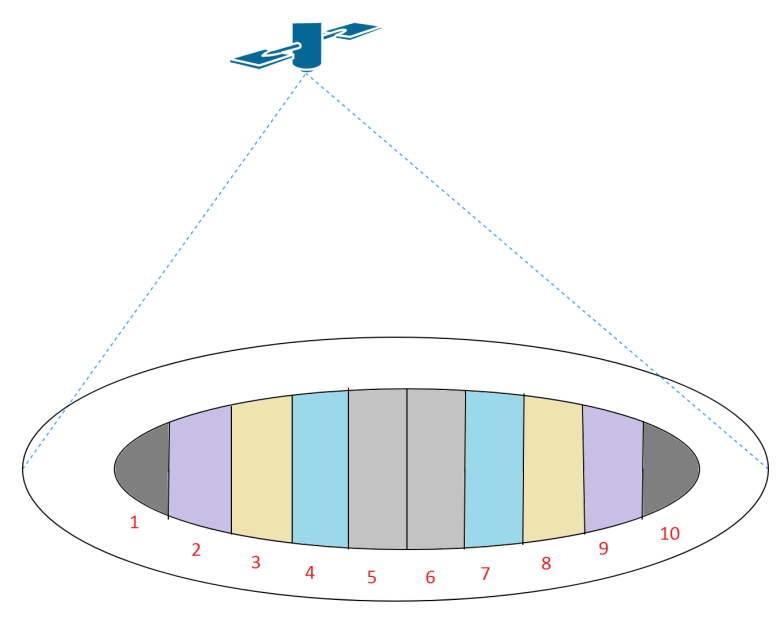

Fig. 8. Coverage area modeling in smaller regions

supported limit along $x$-axis. Furthermore, along $y$-axis the differential Doppler is much lower than the limit, therefore we can consider it as negligible. As a result, the received SCFDMA signal at the eNB will be significantly distorted due to a large amount of overlap between subcarriers (up to $8.5 \mathrm{kHz}$ ), when terminals with large distance in the $x$-axis are scheduled in the same frame.

Of course, if each individual nUE can also pre-compensate its differential Doppler before transmitting the signal, the problem can be solved. In order to do so, the users have to know their location on Earth and estimate the position of the satellite at a certain time continuously. However, this would raise significantly the complexity in the user side. Taking into account the IoT vision of very low cost and low complex devices, alternative solutions should be found, in order to maintain the low complexity of the nUEs.

\section{Proposed Solution}

In order to reduce the high values of differential Doppler in our scenario, we propose a re-modeling of the coverage area in smaller regions in such a way that the differential Doppler inside each region should be below the allowed threshold. It can be easily calculated, by plugging different values of $D$ on equation (11), that at $D=20 \mathrm{~km}$ the maximum differential Doppler along $x$-axis will be below the threshold of $950 \mathrm{~Hz}$. Hence, by dividing the coverage area of $200 \mathrm{~km}$ in 10 smaller regions as shown in Fig. 8, we are certain that inside each region the experienced differential Doppler will be below 950 $\mathrm{Hz}$. Please note that the coverage area is fixed and does not represent the footprint of the satellite, but the area where the nUE are placed on Earth. We have fixed a moment where all the regions are inside the footprint of the satellite. However, during the movement of the satellite it can happen that only some of the regions will be covered with service. In addition, regardless of the position of the satellite, the representation of the regions is fixed (as in fig 8) and does not change with time. It is worth reminding that along the $y$-axis the

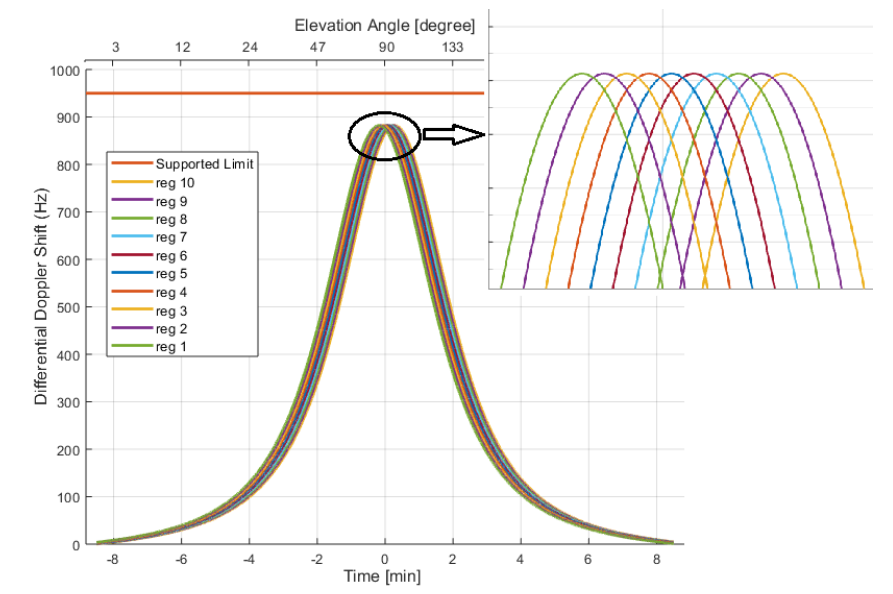

Fig. 9. Maximum differential Doppler angle along $\mathrm{x}$-axis in all the regions

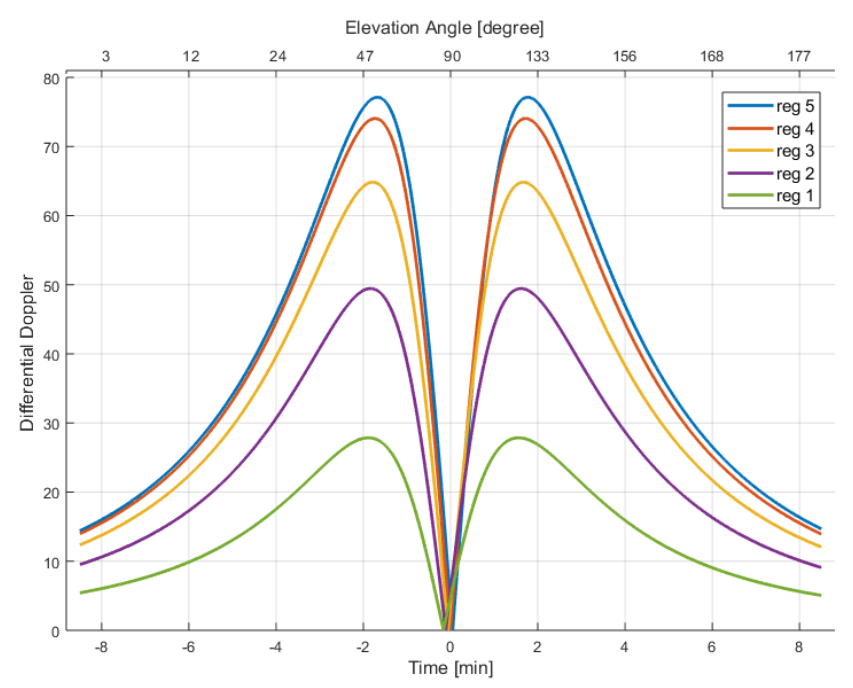

Fig. 10. Maximum differential Doppler angle along y-axis in half of the regions

differential Doppler is so low that smaller regions are not needed in this direction. By performing numerical simulations, we obtain the differential Doppler curves for all the regions along the $x$-axis and $y$-axis as in Fig. 9 and Fig. 10. It can be noted that now the peak values of differential Doppler in each region is approximately $880 \mathrm{~Hz}$ along the $x$-axis, with some small shift in time domain. On the other hand, along the $y$-axis the differential Doppler will be further reduced in some regions, because the maximum distance among 2 nUEs in the $y$-direction will be smaller. The regions with the same colour (see Fig. 8) have the same differential Doppler characteristics and is enough to plot the result only for half of them in the $y$-direction. Again, we have ploted the curves considering elevation angle from 0 to 180 degrees to be more general in our analysis, but the region of interest for each individual curve is from 45 to 135 in our scenario.

Finally, the problem to be solved is how to assign resources in the UL, specifically in the physical uplink shared channel 
(NPUSCH), to all of the re-designed regions. We have identified two ways of assigning the resources.

\section{A. Time Division Multiple Access (TDMA)}

One way of assigning the resources is in the time domain. It means that the eNB assigns the NB-IoT carrier for a certain amount of time to each region. By doing so, the eNB will be able to decode correctly the symbols coming from users placed in the same region no matter how the subcarriers are allocated, as the overlap among them is very small and under the desired limit. Please note that SC-FDMA is still used in the uplink by the nUEs. The drawback of this solution is that the throughput will be reduced by a factor of $N$, where $N$ is the number of regions, which in our case would be 10 . However, in certain applications this is not a problem since the nUEs should report only several times per day to the eNB.

In addition, it is very important to emphasize that the information on what resources to use for each nUE is sent in the downlink control channel NPDCCH. However, the eNB should know the position of all the nUEs, in order to group the users according to the regions and assign the resources properly. As no mobility is foreseen for the nUEs, one possibility is to calibrate the location of each nUE in the deployment phase of the devices in the coverage area. In the Attach Request Procedure, as decribed in [25], the nUEs will identify themselves in the network by sending their IMSI (International Mobile Subscriber Identity) or old GUTI (Globally Unique Temporary ID) and a map can be created which connects their ID with the location in the region. In all the other procedures and message exchanges, the nUE will be identified in the network through this ID, hence the resources can be assigned properly, regardless of the position of the satellite and without increasing any complexity in the user side.

\section{B. Frequency Division Multiple Access (FDMA)}

Another possibility of assigning the uplink resources is in the frequency domain. It means that we have to use several NB-IoT carriers, and not just one, for the uplink transmission. Assigning secondary carriers in the uplink is totally supported by the standard and specified in the Release 14 [26]. However, we should be careful how to spread these carriers along the regions, because now we have to maintain not only the orthogonality between subcarriers inside one NB-IoT carrier, but also among the NB-IoT carriers. There are two ways of doing it:

1) We spread the carriers for each region as shown in Fig. 11. The numbers in red correspond to the number of the region. By doing so, we make sure that the overlap between NB-IoT carriers is minimum because the differential Doppler between adjacent regions is the smallest possible. However, in our scenario, the overlap of two adjacent NB-IoT carriers can pass the limit of 950 $\mathrm{Hz}$ because the distance of two nUE placed in adjacent regions can go from $0-40 \mathrm{~km}$. Due to this reason, we have to double the number of regions which in our case will go to 20 and for each region $D=10 \mathrm{~km}$. By doing so, we maintain the orthogonality under the desired limit, not only inside each NB-IoT carrier but also among adjacent carriers. As a matter of fact, we will need an uplink bandwidth of

$$
B=20 * 180 \mathrm{kHz}=3,6 \mathrm{MHz}
$$

2) We spread the carriers for each region in a random way, but by leaving enough guard band between carriers in such a way that they don't interfere with each other. If we take the case of LTE, this guard band is 1 physical resource block (PRB), which means $180 \mathrm{kHz}$. Therefore, we will need again a bandwidth

$$
B=10 * 180 k H z+10 * 180 k H z=3,6 \mathrm{MHz}
$$

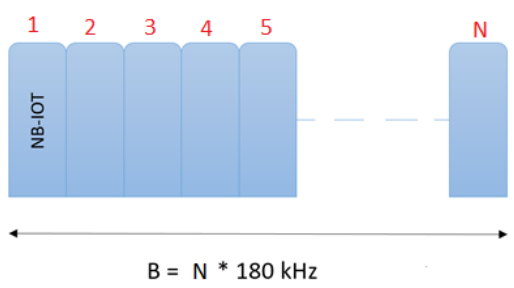

Fig. 11. Needed resources in the uplink transmission

The drawback of FDMA is that we need a lot of secondary carriers, meaning a lot of bandwidth for the NPUSCH. However, with respect to the TDMA solution, it is more efficient in terms of throughput and can be preferred in certain applications of IoT.

\section{CONCLUSIONS AND FUTURE WORK}

In this paper, we considered an NB-IoT over LEO satellite architecture, in order to extend the coverage of terrestrial NBIoT in remote areas and satisfy its goal of ubiquitous global coverage. While assuming perfect estimation and compensation of the common Doppler shift experienced in the satellite channel, we scaled down the problem form the Doppler shift itself to the differential Doppler among nUEs placed in a certain isolated region on Earth. We characterized mathematically the differential Doppler, which in our systems achieves a peak value of approximately $8.5 \mathrm{kHz}$ and reduced it under the supported limit by the standard of $950 \mathrm{~Hz}$, through resource allocation solutions, both in time and in frequency domain.

In the future work, we will consider the scenario where the nUEs can move inside the coverage area. Moreover, a dynamic resource allocation will be studied, in contrast with the one proposed in this paper where we assign the resources based on the peak values of the differential Doppler, even though it changes over time. 


\section{REFERENCES}

[1] W. paper Ericsson, "Cellular networks for massive IoT," January 2016.

[2] A. Ali, W. Hamouda, and M. Uysal, "Next generation M2M cellular networks: challenges and practical considerations," IEEE Communications Magazine, vol. 53, no. 9, pp. 18-24, September 2015.

[3] Y. P. E. Wang, X. Lin, A. Adhikary, A. Grovlen, Y. Sui, Y. Blankenship, J. Bergman, and H. S. Razaghi, "A Primer on 3GPP Narrowband Internet of Things," IEEE Communications Magazine, vol. 55, no. 3, pp. 117123, March 2017.

[4] R. Ratasuk, N. Mangalvedhe, Z. Xiong, M. Robert, and D. Bhatoolaul, "Enhancements of narrowband IoT in 3GPP Rel-14 and Rel-15," in 2017 IEEE Conference on Standards for Communications and Networking (CSCN), Sept 2017, pp. 60-65.

[5] B. Evans, O. Onireti, T. Spathopoulos, and M. A. Imran, "The role of satellites in 5G," in 2015 23rd European Signal Processing Conference (EUSIPCO), Aug 2015, pp. 2756-2760.

[6] F. Bastia et al., "LTE Adaptation for Mobile Broadband Satellite Networks," EURASIP Journal on Wireless Communications and Networking, November 2009.

[7] G. Araniti, M. Condoluci, and A. Petrolino, "Efficient resource allocation for multicast transmissions in Satellite-LTE networks," in 2013 IEEE Global Communications Conference (GLOBECOM), Dec 2013, pp. 3023-3028.

[8] M. Amadeo, G. Araniti, A. Iera, and A. Molinaro, "A Satellite-LTE Network with Delay-Tolerant Capabilities: Design and Performance Evaluation," in 2011 IEEE Vehicular Technology Conference (VTC Fall), Sept 2011, pp. 1-5.

[9] S. Scalise, C. P. Niebla, R. D. Gaudenzi, O. D. R. Herrero, D. Finocchiaro, and A. Arcidiacono, "S-MIM: a novel radio interface for efficient messaging services over satellite," IEEE Communications Magazine, vol. 51, no. 3, pp. 119-125, March 2013.

[10] Riccardo De Gaudenzi et al., "Random access schemes for satellite networks, from VSAT to M2M: a survey," International Journal of Satellite Communications and Networking, December 2016.

[11] 3GPP RP-171578, "Propagation delay and Doppler in Non-Terrestrial Networks," Tech. Rep., September 2017.

[12] 3GPP RP-171453 TR 38.811 V0.1.0, "Study on New Radio (NR) to support Non Terrestrial Networks (Release 15)," Tech. Rep., June 2017.

[13] M. Gineste, T. Deleu, M. Cohen, N. Chuberre, V. Saravanan, V. Frascolla, M. Mueck, E. C. Strinati, and E. Dutkiewicz, "Narrowband IoT Service Provision to 5G User Equipment via a Satellite Component," in 2017 IEEE Globecom Workshops (GC Wkshps), Dec 2017, pp. 1-4.

[14] Z. Qu, G. Zhang, H. Cao, and J. Xie, "LEO Satellite Constellation for Internet of Things," IEEE Access, vol. 5, pp. 18 391-18 401, 2017.

[15] M. D. Sanctis, E. Cianca, G. Araniti, I. Bisio, and R. Prasad, "Satellite Communications Supporting Internet of Remote Things," IEEE Internet of Things Journal, vol. 3, no. 1, pp. 113-123, Feb 2016.

[16] N. Celandroni et al., "A survey of architectures and scenarios in satellite-based wireless sensor networks: system design aspects," International Journal of Satellite Communications and Networking, vol. 31, no. 1, pp. 1-38. [Online]. Available: https://onlinelibrary.wiley.com/doi/abs/10.1002/sat.1019

[17] J. Lin, Z. Hou, Y. Zhou, L. Tian, and J. Shi, "MAP Estimation Based on Doppler Characterization in Broadband and Mobile LEO Satellite Communications," in 2016 IEEE 83rd Vehicular Technology Conference (VTC Spring), May 2016, pp. 1-5.

[18] A. Guidotti, A. Vanelli-Coralli, M. Caus, J. Bas, G. Colavolpe, T. Foggi S. Cioni, A. Modenini, and D. Tarchi, "Satellite-enabled LTE systems in LEO constellations," in 2017 IEEE International Conference on Communications Workshops (ICC Workshops), May 2017, pp. 876-881.

[19] O. Kodheli, A. Guidotti, and A. Vanelli-Coralli, "Integration of Satellites in 5G through LEO Constellations," in GLOBECOM 2017 - 2017 IEEE Global Communications Conference, Dec 2017, pp. 1-6.

[20] J. Li, Y. Zhang, Y. Zhang, W. Xiong, Y. Huang, and Z. Wang, "Fast tracking Doppler compensation for OFDM-based LEO Satellite data transmission," in 2016 2nd IEEE International Conference on Computer and Communications (ICCC), Oct 2016, pp. 1814-1817.

[21] N. Mangalvedhe, R. Ratasuk, and A. Ghosh, "NB-IoT deployment study for low power wide area cellular IoT," in 2016 IEEE 27th Annual International Symposium on Personal, Indoor, and Mobile Radio Communications (PIMRC), Sept 2016, pp. 1-6.
[22] C. Yu, L. Yu, Y. Wu, Y. He, and Q. Lu, "Uplink Scheduling and Link Adaptation for Narrowband Internet of Things Systems," IEEE Access, vol. 5, pp. 1724-1734, 2017.

[23] I. Ali, N. Al-Dhahir, and J. E. Hershey, "Doppler characterization for LEO satellites," IEEE Transactions on Communications, vol. 46, no. 3, pp. 309-313, Mar 1998.

[24] 3GPP TR 25.913, "Requirements for Evolved UTRA (E-UTRA) and Evolved UTRAN (E-UTRAN) (3GPP TR 25.913 version 9.0.0 Release 9)," Tech. Rep., February 2010.

[25] 3GPP TS 24.301, "3rd Generation Partnership Project; Technical Specification Group Core Network and Terminals; Non-Access-Stratum (NAS) protocol for Evolved Packet System (EPS); Stage 3 (Release 13)," Tech. Rep., September 2016.

[26] A. Hoglund, X. Lin, O. Liberg, A. Behravan, E. A. Yavuz, M. V. D. Zee, Y. Sui, T. Tirronen, A. Ratilainen, and D. Eriksson, "Overview of 3GPP Release 14 Enhanced NB-IoT," IEEE Network, vol. 31, no. 6, pp. 16-22, November 2017. 